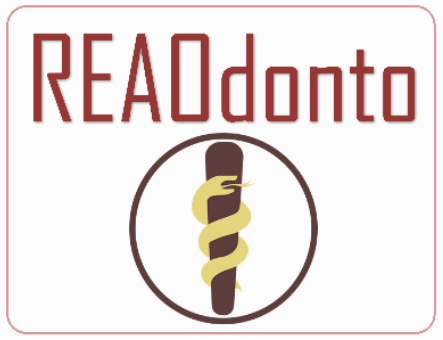

Revista Eletrônica Acervo Odontológico

\section{REVISÃO BIBLIOGRÁFICA}

Recebido em: 12/2020

Aceito em: 12/2020

Publicado em: 2/2021

\title{
Resinas bulkfill: propriedades e aplicabilidades
}

\author{
Bulkfill resins: properties and applicability
}

\section{Resinas a granel: propriedades e aplicabilidades}

Giovanna Garcia Monteiro르. Jenniffer Shayane de Melo Oliveira', Maria Iohanna Brasil Bessa ${ }^{1}$, Zildenilson da Silva Sousa ${ }^{1}$, lana Sá de Oliveira Fontenelle ${ }^{2}$, Juliana Araújo Oliveira², Talita Arrais Daniel Mendes ${ }^{2 *}$.

Resumo: As resinas compostas (RCs) são materiais amplamente utilizados na odontologia como método restaurador. Apesar disso, sua capacidade de espessura possui fatores limitantes que interferem em uma melhora nos resultados. Assim, as RCs bulkfill (RCB) surgem com a possibilidade de incrementos únicos em processos restauradores. O objetivo deste trabalho é revisar a literatura acerca do que se sabe até o atual contexto sobre esse material restaurador, evidenciando a aplicabilidade clínica da RCB, além de suas propriedades como implemento eficaz na odontologia restauradora. Utilizou-se dos descritores em saúde: composite resins, polymerization, operative dentistry, clinical evaluation e chemistry aplicados nas bases de dados PubMed e Science Direct, na língua inglesa e interligados entre si pelo operador booleano "AND". Dos 143 estudos encontrados, 133 foram excluídos por apresentarem um ou mais critérios de exclusão, sendo incluídos 10 estudos nesta revisão. As RCB através de suas propriedades químicas permitem uma maior rapidez em tratamentos clínicos, diminuindo complexibilidades técnicas nos procedimentos profissionais, além de capacidade de incremento mais vantajosa que as RCs convencionais. Contudo, mais estudos com períodos de avaliação mais extensos se fazem necessários antes de uma recomendação definitiva de mudanças de protocolos clínicos.

Palavras-chave: Resinas compostas, Polimerização, Dentística operatória.

\begin{abstract}
Composite resins (RCs) are materials widely used in dentistry as a restorative method. Despite this, its thickness capacity has limiting factors that interfere in an improvement in results. Thus, bulkfill RCs (RCB) arise with the possibility of unique increments in restorative processes. The objective of this work is to review the literature about what is known up to the current context about this restorative material, showing the clinical applicability of RCB, in addition to its properties as an effective implement in restorative dentistry. The following health descriptors were used: composite resins, polymerization, operative dentistry, clinical evaluation and chemistry applied in the PubMed and Science Direct databases, in English and interconnected by the Boolean operator "AND". Of the 143 studies found, 133 were excluded for presenting one or more exclusion criteria, and 10 studies were included in this review. The RCBs through their chemical properties allow greater speed in clinical treatments, reducing technical complexities in professionais procedures, in addition to the ability to increase more advantageous than conventional RCs. However, further studies with longer evaluation periods are needed before a definitive recommendation for changes to clinical protocols.
\end{abstract}

Keywords: Composite resins, Polymerization, Operative dentistry.

Resumen: Las resinas compuestas (CR) son materiales ampliamente utilizados en odontología como método restaurador. Apesar de ello, su capacidad de espesor tiene factores limitantes que interfieren en una mejora

${ }^{1}$ Centro Universitário Mauricio de Nassau (UNINASSAU), Fortaleza - CE.

2 Universidade Federal do Ceará (UFC), Fortaleza - CE. *E-mail: talita_arrais@hotmail.com 
de los resultados. Por lo tanto, los RC de relleno masivo (RCB) surgen con la posibilidad de incrementos únicos en los procesos de restauración. El objetivo de este trabajo es revisar la literatura sobre lo que se conoce hasta el contexto actual sobre este material restaurador, mostrando la aplicabilidad clínica del RCB, además de sus propiedades como un eficaz implemento en odontología restauradora. Se utilizaron los siguientes descriptores de salud: resinas compuestas, polimerización, odontología operativa, evaluación clínica y química aplicadas en las bases de datos PubMed y Science Direct, en inglés e interconectadas por el operador booleano "AND". De los 143 estudios encontrados, 133 se excluyeron por presentar uno o más criterios de exclusión y se incluyeron diez estudios en esta revisión. Los RCB a través de sus propiedades químicas permiten una mayor rapidez en los tratamientos clínicos, reduciendo las complejidades técnicas en los procedimientos profesionales, además de la capacidad de incrementarlos de forma más ventajosa que los RC convencionales. Sin embargo, se necesitan más estudios con períodos de evaluación más largos antes de una recomendación definitiva de cambios en los protocolos clínicos.

Palabras clave: Resinas compuestas, Polimerización, Odontología operatoria.

\section{INTRODUÇÃO}

$\mathrm{Na}$ odontologia restauradora, os materiais compósitos à base de resina permitem um desgaste mínimo da estrutura dentária possibilitando a devolução de estética e função (ERSEN KA, et al., 2019; AKALIN TT, et al., 2018). Essa característica só é possível devido a possibilidade de união ao remanescente dentário devido aos sistemas adesivos, além disso, pode possuir vantagem no tratamento de estrutura dentária enfraquecida (AKALIN TT, et al., 2018; TAHA NA, et al., 2017). Esses materiais dentários restauradores consistem em todos os componentes sintéticos que podem ser utilizados para reparar ou substituir a estrutura dentária (ERSEN KA, et al., 2019).

No entanto, as RCs convencionais exigem uma técnica operatória minuciosa e demorada, possibilitando incrementos de 2 milímetros de espessura, exigindo assim, um grande tempo clínico do profissional (TAHA NA, el al., 2017). Assim, surgiram mudanças nas formulações destas RCs, tais como nas propriedades de contração de polimerização e espessura de incremento, onde deu origem às resinas compostas Bulk-fill (RCB) (BILGILI D, et al., 2020). Essas resinas foram desenvolvidas com o intuito de tornar a realização de restaurações com resinas compostas mais rápida, pois segundo os fabricantes, permitem a fotopolimerização adequada até uma profundidade de 5 milímetros (TOMAZ B, et al., 2016).

Esses materiais possuem diversas vantagens que dentre elas estão a baixa tensão, baixos valores de contração e propriedades mecânicas adequadas, que é de suma importância em procedimentos restauradores de dentes obturados (TAHA NA, et al., 2017). Podem ser aplicados em um incremento de até 5 milímetros, sem a necessidade de cobertura ou nivelamento. As RCB podem ser classificadas quanto à sua viscosidade, em convencionais e fluídas (VELOSO SRM, et al., 2018). Portanto, elas podem ser usadas como base, seguido de cobertura com os compostos de resina convencionais (VELOSO SRM, et al., 2018). Além disso, as resinas bulk possuem a capacidade de aumentar a longevidade clínica da restauração e facilitar seu uso, além do número de incrementos necessários para preencher uma cavidade, que é reduzido em comparação com as técnicas tradicionais incrementais (CHAGAS LS, et al., 2016).

São extremamente promissoras e úteis na clínica visando a restauração de cavidades profundas quando demoradas técnicas não são possíveis devido à baixa adesão do paciente (ZORZIN J, et al., 2014). Apresentam uma menor quantidade de carga do que as RCs convencionais resina micro-híbridas ou nanohíbridas, que requerem a técnica de inserção incremental (AKALIN RZ, et al., 2018). Nesse sentido, essas resinas de preenchimento único e de baixa viscosidade contém uma maior translucidez obtida através da porcentagem reduzida de partículas inorgânicas, além de uma maior quantidade de matriz orgânica, o que Ihe permite apresentar um maior escoamento que proporciona facilidade de manipulação e menor tempo de aplicação (HOLANDA LVB, et al., 2016).

Assim sendo, esses compósitos podem ser considerados uma boa opção restauradora devido ao seu baixo módulo de elasticidade, que absorve as tensões geradas por esses fatores, além disso, outras vantagens relatadas como o manuseio superior, economia de tempo e propriedades de auto adaptação 
(CANALI GD, et al., 2018). Por conseguinte, o material flui facilmente para a cavidade, permitindo facilidade de aplicação e boa adaptação à cavidade das paredes (AKALIN RZ, et al., 2018).

São uma alternativa atraente para restaurações posteriores, no entanto, uma compreensão mais clara do clínico desempenho desta classe relativamente nova de materiais em comparação com os compostos de resina convencionais é necessária (VELOSO SRM, et al., 2018; CANEPELLE TMF, et al., 2016). Através disso, o objetivo desta revisão é descrever, por meio da literatura, a aplicabilidade clínica da RCB na prática odontológica com base em estudos recentes, bem como suas propriedades mecânicas e ópticas.

\section{MÉTODOS}

Para a busca da literatura, utilizou-se dos descritores em saúde cadastrados no Descritores em Ciências da Saúde (DeCS): "composite resins", "polymerization", "operative dentistry", "clinical evaluation" e "chemistry" aplicados nas bases de dados PUBMED e no Science Direct "composite resins" e "clinical evaluation" na língua inglesa e interligados entre si pelo operador booleano "AND". O total de artigos encontrados nos últimos 6 anos (período entre 2014 a 2020) foi de 143 na soma das duas plataformas, sendo que no PUBMED obtevese 131 e no Science Direct 12 resultados.

Os critérios de inclusão foram estudos originais, artigos comparativos, estudos de caso, avaliação clínica e artigos que abordavam todos os descritores aplicados em cada base de dados. Os critérios de exclusão foram artigos incompletos, estudos repetidos, revisões de literatura descritiva, capítulo de livro e que não abordavam o objetivo proposto do estudo. Após estabelecer esses critérios e avaliar criteriosamente os títulos e resumos, foram selecionados e incluídos 10 artigos nesta revisão, sendo que 8 foram do PubMed e 2 do Science Direct. Ademais, utilizou-se do software Microsoft Word 2019 para elaboração do fluxograma metodológico da pesquisa (Figura 1).

Figura 1 - Fluxograma da pesquisa bibliográfica.

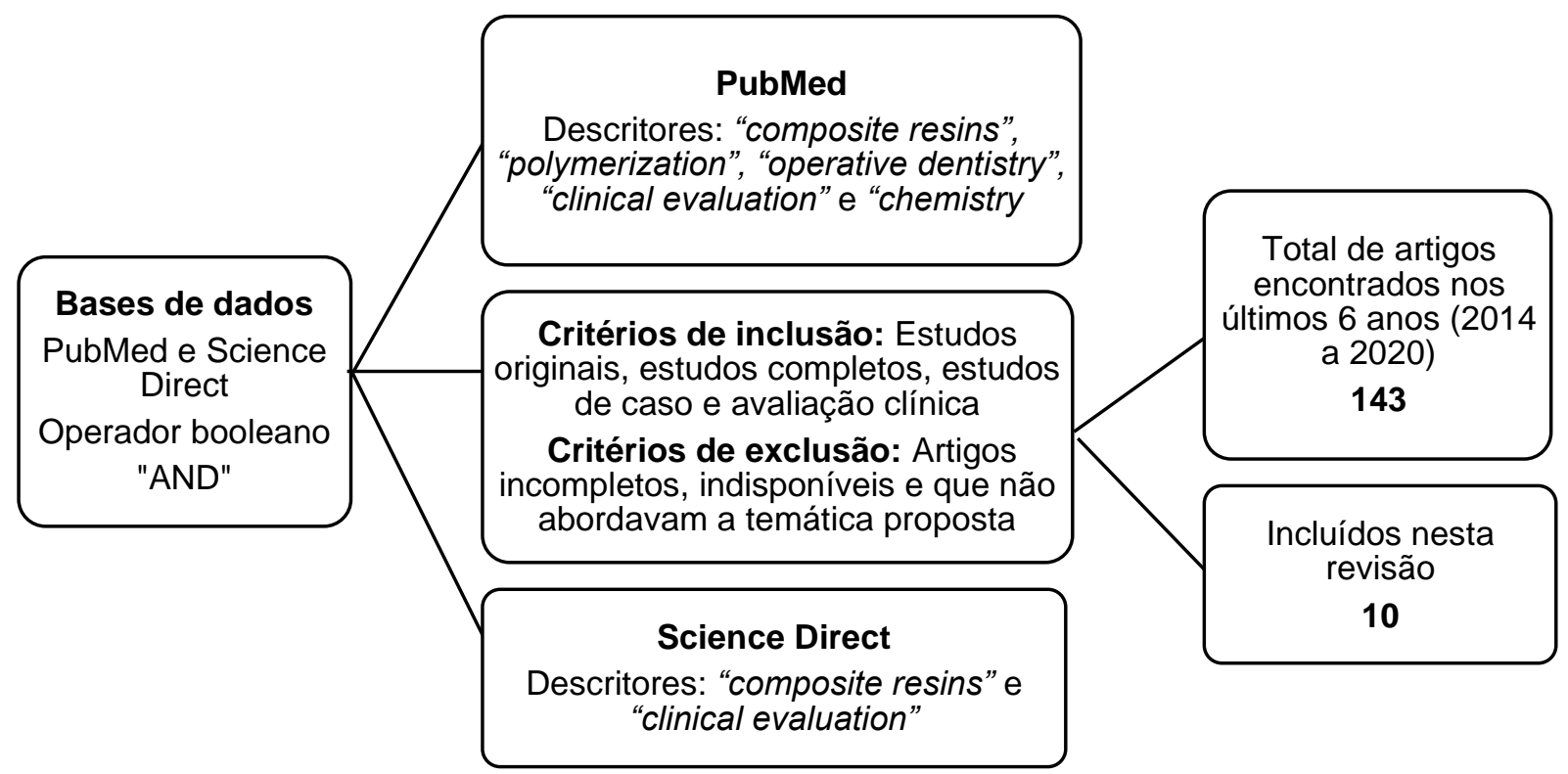

Fonte: Monteiro GG, et al., 2020.

\section{RESULTADOS E DISCUSSÃO}

Após avaliação e discussão dos resultados encontrados em diferentes parâmetros, os dados coletados por meio da literatura foram tabelados visando um melhor entendimento dos estudos obtidos (Tabela 1). Dentre os estudos selecionados estão: 8 ensaios clínicos, 1 estudo randomizado controlado e 1 revisão sistemática com metanálise. 
Tabela 1 - Compilação de dados coletados da literatura por intermédio dos critérios de inclusão e exclusão.

\begin{tabular}{|c|c|c|c|}
\hline Autores/ Ano & Tipo de estudo & Grupos & Resultados \\
\hline $\begin{array}{l}\text { BILGILI D, et al., } \\
2020\end{array}$ & Ensaio clínico & $\begin{array}{l}\text { Sonicfill-2; Filtek Bulk-Fill; } \\
\text { Admira Fusion X-tra; } \\
\text { Bulk Reautifil } \\
\text { controle de vidro }\end{array}$ & 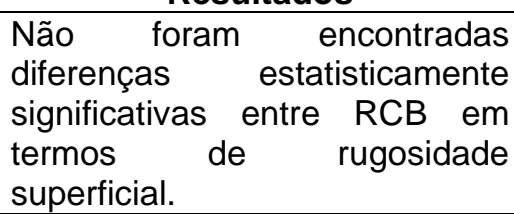 \\
\hline $\begin{array}{l}\text { ERSEN KA, et al., } \\
2019\end{array}$ & Ensaio clínico & $\begin{array}{l}\text { RCB de baixa viscosidade; } \\
\text { RCB alta viscosidade; Filtek } \\
\text { Bulk-Fill; Tetric EvoCeram } \\
\text { Bulk-Fill }\end{array}$ & $\begin{array}{l}\text { Compósitos de RCB mostraram } \\
\text { significativamente menos } \\
\text { contração quando aplicados em } \\
\text { cavidades com resina adesiva e } \\
\text { mais retração após a } \\
\text { polimerização. }\end{array}$ \\
\hline $\begin{array}{l}\text { CANALI GD, et al., } \\
2019\end{array}$ & Ensaio clínico & $\begin{array}{l}\text { Filtek Supreme Ultra Universal } \\
\text { e Filtek Bulk Fill Flowable. }\end{array}$ & $\begin{array}{l}\text { RCB fluidas e RC } \\
\text { nanoparticuladas regulares, } \\
\text { mostraram bons desempenhos } \\
\text { clínicos para a restauração de } \\
\text { Lesões cervicais não cariosas }\end{array}$ \\
\hline $\begin{array}{l}\text { VELOSO SRM, et } \\
\text { al., } 2019\end{array}$ & $\begin{array}{l}\text { Revisão } \\
\text { sistemática } \\
\text { com meta- } \\
\text { análise }\end{array}$ & $\begin{array}{l}\text { Dois revisores independentes } \\
\text { usaram os bancos de dados } \\
\text { PubMed, Embase, The } \\
\text { Cochrane Library e Web of } \\
\text { Science desde o início até } \\
\text { janeiro de 2018. }\end{array}$ & $\begin{array}{l}\text { Desempenhos } \\
\text { semelhantes de RCB e RC } \\
\text { convencionais em um período } \\
\text { de acompanhamento de } 12 \text { a } 72 \\
\text { meses. }\end{array}$ \\
\hline $\begin{array}{c}\text { AKALIN TT, et al., } \\
2018\end{array}$ & Ensaio clínico & $\begin{array}{l}\text { Compósito de RCB nano } \\
\text { híbrido SonicFill; Kerr Corp }\end{array}$ & $\begin{array}{l}\text { RCB mostrou desempenho } \\
\text { clínico aceitável ao longo de } 2 \\
\text { anos de serviço. }\end{array}$ \\
\hline $\begin{array}{l}\text { ALGAMAIAH H, et } \\
\text { al., } 2017\end{array}$ & Ensaio clínico & $\begin{array}{l}\text { Grupo } 1 \text { regular: Vitalescence; } \\
\text { Grupo 2: Resina composta } \\
\text { fluida em massa; Grupo 3: Etric } \\
\text { EvoCeram Bulk;Fill; Gupo 4: } \\
\text { Filtek flowable Bulk Fill }\end{array}$ & $\begin{array}{l}\text { TET exibiu uma contração } \\
\text { volumétrica significativamente } \\
\text { menor em comparação com os } \\
\text { outros compósitos RCB. }\end{array}$ \\
\hline $\begin{array}{l}\text { TAHA NA, et al., } \\
2017\end{array}$ & Ensaio clínico & $\begin{array}{l}\text { Dentes sadios; Dentes não } \\
\text { restaurados; Base Vitrebond e } \\
\text { RC; Base cimento de ionômero } \\
\text { de vidro de } 2 \mathrm{~mm} \text { e laminado } \\
\text { aberto composto à base de } \\
\text { resina; Resina composta fluida } \\
\text { em massa e compósito à base } \\
\text { de resina }\end{array}$ & $\begin{array}{l}\text { A resistência à fratura do grupo } \\
5 \text { RCB foi significativamente } \\
\text { maior do que a resistência à } \\
\text { fratura dos grupos laminados de } \\
\text { cimento de ionômero de vidro }\end{array}$ \\
\hline $\begin{array}{l}\text { VAN D, et al., } \\
2016\end{array}$ & $\begin{array}{l}\text { Estudo } \\
\text { randomizado } \\
\text { controlado }\end{array}$ & $\begin{array}{l}\text { Resina composta fluida em } \\
\text { massa; RC nano-híbrida } \\
\text { (Ceram X mono +); Resina } \\
\text { composta ceram X mono + }\end{array}$ & $\begin{array}{l}\text { A técnica de RCB com redução } \\
\text { de tensões apresentou boa } \\
\text { durabilidade durante o } \\
\text { acompanhamento de } 5 \text { anos. }\end{array}$ \\
\hline $\begin{array}{l}\text { ALSHALI RZ, et } \\
\quad \text { al., } 2015\end{array}$ & Ensaio clínico & $\begin{array}{l}\text { SureFil SDR, Venus Bulk Fill, } \\
\text { base X-tra, Filtek Bulk Fill } \\
\text { fluidos, Sonic Fill, Tetric } \\
\text { EvoCeram Bulk Fill e } \\
\text { Grandioso Flow, Venus } \\
\text { Diamond Flow, X-Flow, Filtek } \\
\text { Supreme XTE, Grandioso, } \\
\text { Venus Diamond, TPH } \\
\text { Spectrum Filtek Z250 }\end{array}$ & $\begin{array}{l}\text { A composição da resina de } \\
\text { compósitos de RCB é } \\
\text { comparável à dos materiais } \\
\text { convencionais, com exceção do } \\
\text { SureFil SDR. }\end{array}$ \\
\hline $\begin{array}{l}\text { ZORZIN J, et al., } \\
2014\end{array}$ & Ensaio clínico & $\begin{array}{l}\text { Filtek BulkFill e Filtek Supreme } \\
\text { XTE Flowable }\end{array}$ & $\begin{array}{l}\text { Compósitos } \mathrm{RCB} \text { obtiveram } \\
\text { propriedades de polimerização } \\
\text { suficientes a } 4 \text { milímetros de } \\
\text { profundidade. }\end{array}$ \\
\hline
\end{tabular}

Fonte: Monteiro GG, et al., 2020. 
As resinas compostas tiveram que passar por algumas alterações, a fim de amenizar algumas desvantagens que o material apresentava (HOLANDA L, et al., 2016). Assim sendo, Bilgili D, et al. (2015) em sua avaliação clínica da bactéria Streptococcus mutans e Streptococcus mitis relacionou a adesão em superfícies de elementos dentários que foram restauradas com compósitos de RCB, os autores evidenciaram que não houve diferença estatisticamente significativa encontrada entre quatro grupos de compostos de enchimento em massa em termos de rugosidade da superfície $(p>0,05)$.

Nessa perceptiva, Ersen KA, et al. (2019) em seu ensaio clínico utilizou uma amostra de 60 terceiros molares extraídos visando avaliar a profundidade de retração de polimerização da cavidade. Os resultados obtidos em seu estudo demonstraram que tipo de material, aplicação de resina adesiva e a profundidade da cavidade afetaram significativamente os valores de redução. Ersen KA, et al. (2019), obteve como desfecho de seu estudo que os compósitos de resina bulk-fill evidenciaram uma menor retração quando utilizados em conjunto com a aplicação de resina adesiva na dentina.

Assim sendo, os estudos de Canali GD, et al. (2019), Veloso SRM, et al. (2019) e Akalin TT, et al. (2018) obtiveram como respostas que as RCB tem uma capacidade de desempenhos clínicos aceitáveis na utilização nas restaurações em elementos dentários, mas que isso pode variar conforme o material, técnica e período em que foi aplicada esses compósitos.

Nesse mesmo sentido, Taha NA, et al. (2017) e Van D, et al. (2017) demonstraram que a aplicação dessas resinas pode ser positiva também na questão da resistência desses materiais. Seus estudos evidenciaram que a técnica de RCB propicia a redução de tensões e apresenta uma boa durabilidade de tempo. Em comparação aos materiais convencionais de restauração, para Alshali RZ, et al. (2015), a composição de compósitos de resina de enchimento a granel é comparável à dos materiais convencionais, com exceção do SureFil SDR.

Com o desenvolvimento dos avanços odontológicos, atualmente tem-se usado a aplicação da nanotecnologia em materiais dentários restauradores diretos, que são as resinas nanoparticuladas (HOLANDA L, et al., 2016). Nesse viés, Zorzin J, et al. (2014) avaliou os efeitos da polimerização e propriedades de fotopolimerização estendida quando aplicadas em resinas compostas, incluindo as RCB. Seu estudo demonstrou que a resina composta condensável convencional Z250 exibiu 38,90\% em espessura de incremento de $2 \mathrm{~mm}$ e apenas $22,20 \%$ na espessura de $4 \mathrm{~mm}$, em comparação com $42,19 \%$ no topo.

Nessa perspectiva, Alshali RZ, et al. (2015) em seu estudo encontrou resultados que mostraram que o monômero variável apresentou diferentes quantidades da RCB e também nos compostos de resinas convencionais. Desse modo, Alshali RZ, et al. (2015) concluiu em seu ensaio clínico que a composição dessas resinas de enchimento a granel foi similar quando comparadas às resinas convencionais como a Filtek Z250, que também foi avaliada no estudo de Zorzin J, et al. (2014) e demais resinas compostas como a TPH Spectrum, Grandioso Flow, Venus Diamond Flow, X-Flow, Filtek Supreme XTE e Venus Diamond.

Assim, para Ersen KA, et al. (2019) os compósitos de resina com altas quantidade de cargas têm menos monômeros e, portanto, menos contração de polimerização, podendo gerar um menor estresse de polimerização na interface de união com o dente. Nesse contexto, Alshali RZ, et al. (2015) evidenciou que muitos monômeros de resina composta como o dimetacrilato de trietilenoglicol (TEGDMA) podem atuar como sensibilizadores que induzem vários tipos de reações alérgicas como as ulcerações, líquen plano e dermatite de contato. Nessa concepção, a solução para reduzir o estresse de contração de polimerização em bulk-fills fluidos, segundo Zorzin J, et al. (2014) pode ser encontrada na redução da quantidade de monômeros de baixo peso molecular como o (TEGDMA) a fim de manter a viscosidade baixa sem aumentar a quantidade do mesmo, sendo que monômeros de alto peso molecular como Urenano Dimetacrilato (UDMA) e dimetacrilatos etoxilados (Bis-EMA) também são usados.

A ausência de fortes interações secundárias intermoleculares, como pontes de hidrogêneo, além da baixa viscosidade e da alta flexibilidade conferem ao TEGDMA os mais altos valores de conversão monomérica (JUNIOR ECR, 2015). As vantagens do bisfenol glicidil metacrilato (BISGMA) era seu peso molecular superior e uma contração de polimerização inferior que o metil metacrilato (CHAGAS LS, et al., 2016). 
Nesse mesmo contexto, Zorzin J, et al. (2014) avaliou cinco compósitos bulk-fill disponíveis comercialmente: Filtek Bulk Fill Restorative, SDR Surefil, Tetric EvoCeram Bulk Fill, Venus Bulk Fill e X-tra Base, além de um composto fluido convencional: Filtek Supreme XTE Flow e um composto condensável Z250. Com o estudo, Zonzin J, et al. (2014) concluiu que a espessura do incremento não tem influência significativa no grau de conversão ou dureza de compósitos de resina bulk-fill e convencionais. Desse modo, os polímeros em geral podem ter sua composição modificado para adequar as demandas específicas, compreendendo o impacto de tais alterações no encolhimento por meio de um método preciso e confiável capaz de quantificar e imagens as mudanças de volume são de grande interesse (HIRATA R, et al., 2015).

Para Algamaiah H, et al. (2016) a contração volumétrica dos compósitos Tetric EvoCeram Bulk Fill, Vitalescence, SureFil SDR Flow, Filtek flowable Bulk Fill, não tiveram resultados significativos para causar danos significativos em cavidades, porém houve diferentes padrões de encolhimentos. Adicionalmente, o estudo de Ersen KA, et al. (2019) evidencia que todos os compósitos bulk-fill apresentaram contração de polimerização, e esse achado variou dependendo do fabricante, pois a perda volumétrica foi significativamente maior em SDR e FB em comparação com TB.

Complementando, Taha NA, et al. (2017) em seu ensaio clínico com cinquenta dentes pré-molares superiores evidenciou que o uso de base composta fluida de bulk-fill (SDR) melhorou significativamente a resistência à fratura resistência dos dentes obturados em comparação com outras restaurações laminadas e dentes não restaurados, no entanto, não melhorou os padrões de fratura para materiais. Complementando, Taha NA, et al. (2017) obteve como resultado de seu estudo que a restauração em geral aumentou a resistência à fratura em comparação com os dentes não restaurados.

Nesse mesmo contexto, Canali GD, et al. (2018) avaliou o desempenho clínico de um compósito bulk fill fazendo o comparativo com o compósito nano preenchido. Ambos os compósitos obtiveram resultados aceitáveis no estudo feito pelo Canali GD, et al. (2018), entretanto houve algumas alterações no caso da rugosidade da superfície, na adaptação marginal e na forma anatômica, sendo que essas alterações ocorreram após 1 ano, mas que segundo Canali GD, et al. (2018) através da relevância clínica de seu estudo as RCB apresentam bons desempenhos clínicos após 1 ano.

Nesse viés, Ersen KA, et al. (2019) através de seu ensaio clínico avaliou a influência da profundidade da cavidade e o uso de resina adesiva na dentina na contração de polimerização de compósitos de resina bulkfill e observou fatores como o tipo de material, a aplicação de resina adesiva e a profundidade da cavidade podem afetar significativamente os valores de contração, além de que em seu estudo todos os compósitos de RCB testados mostraram significativamente menos encolhimento quando aplicados em cavidades com resina adesiva.

Corroborando com Akalin TT, et al. (2018) também se utilizou da profundidade de polimerização em vários compostos de RCB e notou que a durabilidade do material testado foi clinicamente aceitável no seguimento de 2 anos. Assim, este material de preenchimento a granel pode ser indicado para grandes cavidades com resultados aceitáveis, e suas deteriorações significativas em seu estudo podem ser atribuídas à possibilidade do material.

Bilgili D, et al. (2020) avaliou a rugosidade da superfície, energia livre de superfície (SFE) e ângulo de contato de compósitos bulk-fill, bem como a adesão de bactérias Streptococcus mutans e Streptococcus mitis a esses compósitos, sendo que em seu ensaio clínico não houve diferenças significativas em compósitos nanohíbridos, nanofill e bulk-fill em termos de rugosidade da superfície. No entanto, os autores afirmaram que o compósito nano preenchimento exibiu menor adesão bacteriana do que o compósito bulk-fill e que a formação de biofilme de Streptococcus mutans foi significativamente afetada pela composição do material, mas não foi afetada pelos procedimentos de acabamento e polimento.

Van D, et al. (2016) em seu ensaio clínico de um período de 5 anos avaliou a durabilidade das restaurações as quais foram empregadas com a técnica de Bulk-Fill e evidenciou que essa tem um desempenho clinicamente aceitável. Assim sendo, em seu estudo o grupo de RCB apresentou taxa de falha anual de 1,4\%, enquanto no grupo das restaurações feitas com resinas compostas convencionais apresentou taxa de $2,1 \%$. 
Além disso, nenhuma diferença estatística foi observada em restaurações feitas com ou sem reposição inteligente de dentina.

O estudo de Van D, et al. (2016) utilizou de restaurações feitas com resinas compostas Ceram X em Classe II, colocadas com um adesivo autocolante de uma etapa, o antecessor Xeno V, quando comparadas com restaurações coladas com um adesivo Etch-And-Rinse de duas etapas obtiveram uma taxa de falha anual foi semelhante de $1,8 \%$ e $1,2 \%$ para ambos os grupos de adesivos. O resultado obtido no estudo de Van D, et al. (2016) mostraram evidências clínicas claras de que o uso de resina composta mais espessa, incrementadas com o composto fluido resultaram em durabilidade aceitável, que pode economizar tempo de cura, mas também pode ter vantagens em várias situações clínicas como cavidades profundas e outras localizações de difícil acesso com a unidade de cura.

Veloso SRM, et al. (2019) em seu ensaio clínico evidenciou que o desempenho dos compósitos de RCB é comparável às resinas convencionais em restaurações posteriores diretas. A maior translucidez dos compósitos de RCB e o uso de foto iniciadores mais reativos permitem uma maior profundidade de cura e a maior reatividade permite a inserção do material em incrementos de espessura de 4-5 mm, com polimerização e grau de conversão uniformes. (VELOSO SRM, et al., 2019).

Portanto, Franca S (2016) destaca que a dentística foca em princípios de prevenção na odontologia restauradora, contribuindo assim para a utilização de técnicas minimamente invasivas que auxiliam na redução de perdas de tecidos dentário saudáveis, além da utilização de materiais que visem a redução do tempo clínico de tratamento.

\section{CONSIDERAÇÕES FINAIS}

As RCB demonstram ter uma boa aplicabilidade clínica, sendo eficaz em procedimentos estéticos e na reabilitação da funcionalidade do elemento dentário, restaurando a saúde oral. Suas propriedades químicas permitem uma maior rapidez em tratamentos clínicos, diminuindo complexibilidades técnicas nos procedimentos profissionais, além de se ter uma capacidade de incremento mais vantajosa que as resinas compostas convencionais, reduzindo os efeitos adversos da contração de polimerização do material. Apesar das evidências comprovarem a aplicabilidade deste material restaurador, mais estudos com períodos de avaliação mais extensos ainda se fazem necessários e importantes antes de uma recomendação definitiva de mudanças de protocolos clínicos em relação a essas resinas compostas de baixa contração.

\section{AGRADECIMENTOS}

Ao Centro Universitário Maurício de Nassau.

\section{REFERÊNCIAS}

1. ALSHALI RZ, et al. Qualitative and quantitative characterization of monomers of uncured bulk-fill and conventional resin-composites using liquid chromatography/mass spectrometry. Dent Mater. 2015; 31(6): 711-720. 14.

2. ALGAMAIAH H, et al. Microcomputed Tomography Evaluation of Volumetric Shrinkage of Bulk-Fill Composites in Class II Cavities. J Esthet Restor Dent. 2017; 29(2): 118-127.

3. AKALIN TT, et al. Clinical Evaluation of Sonic-Activated High Viscosity Bulk-Fill Nanohybrid Resin Composite Restorations in Class II Cavities: A Prospective Clinical Study up to 2 Years. Eur J Prosthodont Restor Dent. 2018; 26(3): 152-160.

4. BILGILI D, et al. Surface properties and bacterial adhesion of bulk-fill composite resins. J Dent., 2020; 95 : 103317.

5. CANALI GD, et al. One-year clinical evaluation of bulk-fill flowable vs. regular nanofilled composite in non-carious cervical lesions. Clin Oral Investig. 2019; 23(2): 889-897.

6. CANEPPELE TMF, BRESCIANI E. Resinas bulk-fill - O estado da arte. Rev. Assoc. Paul. Cir. Dent. [online]. 2016; 70(3): 242-248.

7. CHAGAS LS. Avaliação da resistência flexural e módulo de elasticidade de uma resina do tipo bulk fill em comparação com uma resina convencional, 2016.

8. ERSEN KA, et al. Evaluation of polymerization shrinkage of bulk-fill resin composites using microcomputed tomography. Clin Oral Investig. 2020; 24(5): 1687-1693.

9. FRANCA S. Odontologia restauradora na era adesiva. Rev. Assoc. Paul. Cir. Dent. [online]. 2016; 70(3): $234-241$. 
10. HIRATA R, et al. Shrinkage assessment of low shrinkage composites using microcomputed tomography. J Biomed Mater Res B Appl Biomater, 2015; 103(4): 798-806.

11. HOLANDA LVB, et al. Desempenho das propriedades físico-mecânicas das resinas bulk-fill: revisão de literatura. Jornada Odontológica dos Acadêmicos da Católica, 2016; 2(1).

12. JUNIOR ECR. Estudo de propriedades de resinas compostas bulk fill. 2015. Dissertação de Mestrado (Odontologia) - USP, 2016.

13. TAHA NA, et al. Effect of bulk-fill base material on fracture strength of root-filled teeth restored with laminate resin composite restorations. J Dent. 2017; 63: 60-64.

14. TOMAZ B, et al. Análise da microdureza de resinas compostas bulk fill. In: Encontros Universitários da UFC, 2016; 1

15. VAN D, et al. Posterior bulk-filled resin composite restorations: A 5-year randomized controlled clinical study. Journal of Dentistry, 2016; 51: 29-35.

16. VELOSO SRM, et al. Clinical performance of bulk-fill and conventional resin composite restorations in posterior teeth: a systematic review and meta-analysis. Clin Oral Investig. 2019; 23(1): 221-233.

17. ZORZIN J, et al. Bulk-fill resin composites: polymerization properties and extended light curing. Dent Mater. 2015; 31(3): 293-301. 